\title{
Discharge Before Return to Respiratory Baseline in Children With Neurologic Impairment
}

\author{
Rebecca Steuart, MD¹, Rachel Tan, BS², Katherine Melink, BS², Sofia Chinchilla, BS², \\ Amanda Warniment, MD³, Samir S Shah, MD, MSCE ${ }^{1,4}$, Joanna Thomson, MD, MPH ${ }^{1,4 *}$
}

\begin{abstract}
${ }^{1}$ Division of Hospital Medicine, Cincinnati Children's Hospital Medical Center, Cincinnati, Ohio; ${ }^{2}$ University of Cincinnati College of Medicine, Cincinnati, Ohio; ${ }^{3}$ Pediatrics Housestaff, Cincinnati Children's Hospital Medical Center, Cincinnati, Ohio; ${ }^{4}$ Department of Pediatrics, University of Cincinnati, Cincinnati, Ohio.
\end{abstract}

BACKGROUND: Children with neurologic impairment (NI) are commonly hospitalized with acute respiratory infections (ARI). These children frequently require respiratory support at baseline and are often discharged before return to respiratory baseline.

OBJECTIVE: To determine if discharge before return to respiratory baseline is associated with reutilization among children with NI hospitalized with ARI.

METHODS: This single-center retrospective cohort study included children with $\mathrm{NI}$ aged 1 to 18 years hospitalized with ARI who required increased respiratory support between January 2010 and September 2015. The primary exposure was discharge before return to respiratory baseline. The primary outcome was 30-day hospital reutilization. A generalized estimating equation was used to examine the association between exposure and outcome while accounting for within-patient clustering and patient-level clinical complexity and illness severity.
RESULTS: In the 632 hospitalizations experienced by 366 children, children were discharged before return to respiratory baseline in $30.4 \%$ of hospitalizations. Compared with those hospitalizations in which children were discharged at baseline, hospitalizations with a discharge before return to respiratory baseline were more likely to be for privately insured, technology-dependent children with respiratory comorbidities. Compared with discharges at respiratory baseline, discharges with increased respiratory support had no difference in 30-day reutilization (32.8\% vs $31.8 \%$; $P=.81$; adjusted OR 0.80 , 95\% Cl 0.51-1.26).

CONCLUSIONS: Among children with NI hospitalized with ARI, discharge before return to respiratory baseline was common, but it was not associated with hospital reutilization. Return to respiratory baseline may not be a necessary component of discharge criteria in this population. Journal of Hospital Medicine 2020; 15:531-537. (c) 2020 Society of Hospital Medicine hildren with neurologic impairment (NI; eg, hypoxicischemic encephalopathy, muscular dystrophy) are characterized by functional and/or intellectual impairments resulting from a variety of neurologic diseases. ${ }^{1}$ These children commonly have respiratory comorbidities, including central hypoventilation, impaired cough, and oromotor dysfunction, that may lead to chronic respiratory insufficiency and a need for chronic respiratory support at baseline., Baseline respiratory support modalities can include supplemental oxygen, noninvasive positive pressure ventilation, or invasive mechanical ventilation.

Acute respiratory infections (ARl; eg, pneumonia, bronchiolitis) are the most common cause of hospitalization, intensive

*Corresponding Author: Joanna Thomson, MD, MPH;

Email: joanna.thomson@cchmc.org; Telephone: 513-636-0257;

Twitter: @JoThomsonMD.

Published online first May 20, 2020.

Find additional supporting information in the online version of this article.

Received: October 7, 2019; Revised: February 4, 2020;

Accepted: February 10, 2020

(c) 2020 Society of Hospital Medicine DOI 10.12788/jhm.3394 care unit (ICU) admission, and death for children with NI., ${ }^{1,3}$ Discharge criteria for otherwise healthy children admitted to the hospital with ARI often include return to respiratory baseline. ${ }^{4}$ Children with complex chronic conditions have longer hospitalizations when hospitalized with respiratory infections, ${ }^{5-7}$ because, in part, comorbidities and complications prolong the time to return to baseline. This prolonged return to respiratory baseline in combination with family knowledge, comfort, and skill in managing respiratory support and other complexities at home may alter discharge practices in the population of children with NI. In our clinical experience, discharge before return to baseline respiratory support occurs more frequently in children with $\mathrm{NI}$ than in otherwise healthy children when hospitalized with ARI. However, the consequences of discharging children with $\mathrm{NI}$ prior to return to respiratory baseline are unknown.

In this study, we sought to determine if discharge prior to return to baseline respiratory support is associated with reutilization among children with $\mathrm{NI}$ hospitalized with ARI. We hypothesized that patients discharged prior to return to respiratory baseline would have higher rates of 30-day hospital reutilization. 


\section{METHODS}

\section{Study Design and Data Source}

This single-center, retrospective cohort study of children hospitalized at Cincinnati Children's Hospital Medical Center (CCHMC) used data from the Pediatric Health Information System (PHIS) and the electronic medical record (EMR). PHIS, an administrative database of 45 not-for-profit, tertiary care, US pediatric hospitals managed by Children's Hospital Association (Lenexa, Kansas), was used to identify eligible children, examine demographic and clinical variables, and define outcomes. PHIS contains data regarding patient demographics, inpatient resource utilization, and diagnoses. Encrypted medical record numbers in PHIS allowed for local identification of patients' medical records to complete EMR review to confirm eligibility and obtain detailed patient-level clinical information (eg, respiratory support needs) not available in PHIS.

Pilot medical record reviews allowed for standardized study definitions and procedures. All study staff underwent training with the primary investigator, including detailed review of 10 initial abstractions. Two investigators (K.M. and S.C.) performed repeat abstractions from 40 randomly selected records to enable assessment of interrater reliability. Average reliability, indicated by the $\kappa$ statistic, indicated substantial to near-perfect reliability ${ }^{8}(\kappa=0.97,95 \% \mathrm{Cl} 0.90-1.00)$ for the primary exposure. EMR data were managed using Research Electronic Data Capture (REDCap, Nashville, Tennessee) ${ }^{9}$ and subsequently merged with PHIS data.

\section{Study Population}

Hospitalizations of children with $\mathrm{NI}$ aged 1 to 18 years at CCHMC between January 2010 and September 2015 were eligible for inclusion if they had a principal discharge diagnosis indicative of $A R I$ and required increased respiratory support from baseline during hospitalization. NI was defined as a high-intensity, chronic neurological diagnosis with substantial functional impairments according to previously defined diagnosis codes. ${ }^{1,10}$ ARI was identified using codes in the Clinical Classification Software (Agency for Healthcare Research and Quality, Rockville, MD) respiratory group indicative of ARI (eg, pneumonia, bronchiolitis, influenza; Appendix Table).

Children transferred to CCHMC were excluded because records from their initial illness presentation and management were not available. Because of expected differences in management and outcomes, children with a known diagnosis of tuberculosis or human immunodeficiency virus were excluded. Because exposure criteria were dependent on hospital discharge status, hospitalizations for children who died during admission (4 of 632 hospitalizations, $0.63 \%$ ) were excluded from the final cohort (Appendix Figure).

\section{Study Definitions}

Baseline respiratory support (ie, "respiratory baseline") was defined as the child's highest level of respiratory support needed prior to admission when well (ie, no support, supplemental oxygen, continuous positive airway pressure [CPAP] or bilevel positive airway pressure [BiPAP], or ventilator support), and further characterized by night or day/night requirement. Respiratory baseline was identified using EMR documentation of home respiratory support use at the time of index admission. Return to respiratory baseline was defined as the date on which the child achieved documented home respiratory support settings, regardless of clinical symptoms.

Children may have required increased respiratory support from baseline at any time during hospitalization. Maximum respiratory support required was categorized as one of the following: (1) initiation of supplemental oxygen or increase in oxygen flow or duration; (2) initiation of CPAP or BiPAP; (3) increase in pressure settings or duration of pressure support for those with baseline CPAP, BiPAP, or ventilator use; and (4) initiation of full mechanical ventilation. Respiratory support categories were mutually exclusive: children requiring multiple types of increased respiratory support were classified for analysis by the most invasive form of respiratory support used (eg, a child requiring increase in both oxygen flow and pressure settings was categorized as an increase in pressure settings). Children who received heated high-flow nasal cannula therapy as maximum support were categorized as initiation or increase in oxygen support.

Time to return to respiratory baseline was defined as the difference in days between date of return to respiratory baseline and date of admission. Time to return to respiratory baseline was determined only for children who were discharged at respiratory baseline.

\section{Primary Exposure and Outcome Measures}

The primary exposure was hospital discharge before return to respiratory baseline (ie, discharge on higher respiratory support than at baseline settings). At our institution, standardized discharge criteria for children with $\mathrm{NI}$ do not exist. The primary outcome was all-cause, 30-day hospital reutilization, including hospital readmissions and emergency department (ED) revisits. Secondary outcomes included 30-day reutilization for ARI and hospital length of stay (LOS) in days.

\section{Patient Demographics and Clinical Characteristics}

Demographic and patient characteristics that might influence hospital discharge before return to respiratory baseline or readmission were obtained from PHIS (eg, demographic information, age, insurance type, measures of clinical complexity, illness severity) and by EMR review (eg, baseline respiratory support needs, maximum respiratory support during hospitalization). Measures of clinical complexity included comorbid complex chronic conditions (CCCs) ${ }^{11-14}$ and technology dependence ${ }^{14-16}$ using previously defined diagnostic codes. Measures of illness severity included sepsis ${ }^{17}$ and ICU-level care. At our institution, children with baseline ventilator use do not require admission to the ICU unless they are clinically unstable.

\section{Statistical Analysis}

Continuous variables were described using medians and interquartile ranges (IQR). Categorical variables were described using counts and percentages. Patient characteristics and outcomes were stratified by primary exposure and compared us- 
ing chi-square test or Fisher exact test for categorical variables and Wilcoxon rank sum test for continuous variables.

To examine the independent association between discharge before return to respiratory baseline and hospital reutilization, a generalized estimating equation was used that included potential confounders while accounting for within-patient clustering. Patient demographics included age, race, ethnicity, and insurance type; measures of clinical complexity included number of CCCs, technology dependence, and baseline respiratory support; and measures of acute illness severity included ARI diagnosis, degree of increase in respiratory support during hospitalization, and ICU-level care. LOS was also included in the model as a covariate because of its expected association with both exposure and outcome.

Secondary analyses were conducted using the outcome of 30-day reutilization for ARI. Subgroup analysis excluding hospitalizations of children lost to follow-up (ie, no encounters in the 6 months after hospital discharge) was also conducted. All analyses were performed with SAS v9.3 (SAS Institute, Cary, North Carolina). $P$ values $<.05$ were considered statistically significant. This study was approved by the Institutional Review Board.

\section{RESULTS}

\section{Study Cohort}

A total of 632 hospitalizations experienced by 366 children with $\mathrm{NI}$ who were admitted with ARI were included (Appendix Figure). Most children (66.4\%) in the cohort experienced only one hospitalization, $17.5 \%$ had two hospitalizations, $7.9 \%$ had three hospitalizations, and $8.2 \%$ had four or more hospitalizations. The median age at hospitalization was 5.0 years (IOR 2.8-10.5) and most hospitalizations were for children who were male $(56.6 \%)$, white (78.3\%), non-Hispanic (96.0\%), and publicly insured (51.7\%; Table 1). More than one-quarter (28.6\%) of hospitalizations were for children with four or more CCCs, and in $73.4 \%$ of hospitalizations, children were technology dependent (Table 1). Baseline respiratory support was common (46.8\%), including home mechanical ventilation in $11.1 \%$ of hospitalizations (Table 1). Bacterial pneumonia, including aspiration pneumonia, was the most common discharge diagnosis (50.5\%, Table 1$)$.

\section{Demographic and Clinical Characteristics}

Children were discharged before return to respiratory baseline in $30.4 \%$ of hospitalizations (Appendix Figure). Children discharged before return to respiratory baseline were older (median age 5.7 years, IOR 3.1-11.0, vs 4.9 years, IOR 2.6$9.7 ; P=.04)$ and more likely to be privately insured $(54.2 \%$ vs $43.4 \% ; P=.04)$, compared with children discharged at respiratory baseline (Table 1). Children discharged before return to respiratory baseline were also more likely to have a respiratory CCC (59.9\% vs 30.9\%; $P<.001)$, have a respiratory technology dependence diagnosis code (44.8\% vs $24.1 \%$; $P<.001$ ), and have baseline respiratory support needs on EMR review $(67.7 \%$ vs $37.7 \% ; P<.001)$, compared with children discharged at baseline (Table 1).

Children discharged before return to respiratory baseline required significantly greater escalation in respiratory support during hospitalization, compared with children discharged at respiratory baseline, including higher rates of initiation of CPAP or BiPAP, increased pressure settings from baseline (for home CPAP, BiPAP, or ventilator users), and initiation of full mechanical ventilation (Table 1). Hospitalizations in which children were discharged before return to respiratory baseline were also more likely to include ICU care than were those for children discharged at baseline (52.1\% vs $35.2 \%$; $P<.001$; Table 1 ).

\section{Clinical Outcomes and Utilization}

Reutilization within 30 days occurred after $32.1 \%$ of hospitalizations, with $26.1 \%$ requiring hospital readmission and $6.0 \%$ requiring ED revisit (Table 2 ). There was no statistical association in either unadjusted (Table 2) or adjusted (Table 3) analysis between children discharged before return to respiratory baseline and 30-day all-cause hospital reutilizations, hospital readmissions, or ED revisits.

In analysis of secondary outcomes, 30-day reutilization because of ARI occurred after $21.5 \%$ of hospitalizations, with $19.0 \%$ requiring hospital readmission and $2.5 \%$ requiring $E D$ revisit. Median hospital LOS for the cohort was 4 days (IQR 2-8; Table 2). Hospitalizations in which children were discharged before return to respiratory baseline were longer than in those discharged at baseline (median 6 days, IOR 3-11, vs 4 days, IQR 2-7; $P<.001$; Table 2).

For hospitalizations of children discharged at respiratory baseline, the median time to return to respiratory baseline was 3 days (IQR 1-5, complete range 0-80). In these encounters, discharge occurred soon after return to respiratory baseline (median 1 day, IOR 0-1.5, complete range 0-54).

In subgroup analysis excluding the 18 hospitalizations in which children were lost to follow-up (2.8\% of the total cohort), discharge before return to respiratory baseline was not associated with 30-day all-cause hospital reutilization (Table 4).

\section{DISCUSSION}

In this retrospective cohort study, children with NI hospitalized with ARI were frequently discharged using increased respiratory support from baseline. However, those discharged before return to respiratory baseline, despite their greater clinical complexity and acute illness severity, did not have increased hospital reutilization, compared with children discharged at respiratory baseline. Our findings suggest that discharge before return to baseline respiratory support after ARI may be clinically appropriate in some children with NI.

With the growing emphasis on decreasing hospital costs, concern exists that patients are being discharged from hospitals "quicker and sicker," 18,19 with shortening lengths of stay and higher patient instability at discharge. Clinical instability at discharge has been associated with adverse postdischarge outcomes in adults with pneumonia ${ }^{20-23}$; however, studies evaluating discharge readiness have not examined the population of children with NI. Our findings of no difference in reutilization for children with $\mathrm{NI}$ discharged before return to respiratory baseline, which would be expected to approximate one or more clinical instabilities, contrast these concerns. 
TABLE 1. Cohort Clinical Characteristics and Hospital Course

\begin{tabular}{|c|c|c|c|c|c|c|c|}
\hline Demographics & \multicolumn{2}{|c|}{$\begin{array}{c}\text { Overall } \\
(\mathrm{N}=632)\end{array}$} & \multicolumn{2}{|c|}{$\begin{array}{l}\text { Discharged before return } \\
\text { to respiratory baseline } \\
\qquad(n=192)\end{array}$} & \multicolumn{2}{|c|}{$\begin{array}{l}\text { Discharged at } \\
\text { respiratory baseline } \\
(n=440)\end{array}$} & $P$ value $^{\mathrm{a}}$ \\
\hline Age in years (median, [IQR]) & 5.0 & $(2.8,10.5)$ & 5.7 & $(3.1,11.0)$ & 4.9 & $(2.6,9.7)$ & .04 \\
\hline Male gender & 358 & $(56.6)$ & 115 & $(59.9)$ & 243 & $(55.2)$ & .28 \\
\hline White & 495 & $(78.3)$ & 161 & (83.9) & 334 & $(75.9)$ & \\
\hline Black & 88 & $(13.9)$ & 18 & (9.4) & 70 & $(15.9)$ & \\
\hline Other & 49 & $(7.8)$ & 13 & (6.8) & 36 & (8.2) & \\
\hline Hispanic ethnicity & 25 & $(4.0)$ & 5 & (2.6) & 20 & (4.5) & .37 \\
\hline Insurance type & & & & & & & .04 \\
\hline
\end{tabular}

\section{Clinical Characteristics}

\begin{tabular}{|c|c|c|c|c|c|c|c|}
\hline \multicolumn{8}{|l|}{ Type of complex chronic conditions ${ }^{b}$} \\
\hline Neuromuscular & 442 & $(69.9)$ & 150 & (78.1) & 292 & (66.4) & .003 \\
\hline Gastrointestinal & 420 & $(66.5)$ & 149 & $(77.6)$ & 271 & (61.6) & $<.001$ \\
\hline Genetic & 346 & $(54.7)$ & 101 & $(52.6)$ & 245 & $(55.7)$ & .47 \\
\hline Respiratory & 251 & (39.7) & 115 & $(59.9)$ & 136 & (30.9) & $<.001$ \\
\hline Technology dependence ${ }^{b}$ & 464 & $(73.4)$ & 162 & $(84.4)$ & 302 & $(68.6)$ & $<.001$ \\
\hline Gastrointestinal & 414 & $(65.5)$ & 148 & $(77.1)$ & 266 & $(60.5)$ & $<.001$ \\
\hline Respiratory & 192 & $(30.4)$ & 86 & $(44.8)$ & 106 & (24.1) & $<.001$ \\
\hline Neurologic & 90 & $(14.2)$ & 49 & (25.5) & 41 & $(9.3)$ & $<.001$ \\
\hline \multicolumn{7}{|l|}{ Baseline respiratory support ${ }^{c}$} & $<.001$ \\
\hline None & 336 & $(53.2)$ & 62 & $(32.3)$ & 274 & $(62.3)$ & \\
\hline Oxygen & 122 & $(19.3)$ & 43 & (22.4) & 79 & $(18.0)$ & \\
\hline CPAP/BIPAP & 104 & (16.4) & 62 & $(32.3)$ & 42 & $(9.5)$ & \\
\hline Ventilator & 70 & $(11.1)$ & 25 & $(13.0)$ & 45 & $(10.2)$ & \\
\hline \multicolumn{7}{|l|}{ Acute respiratory infection (ARI) type } & .004 \\
\hline Pneumonia & 319 & $(50.5)$ & 90 & $(46.9)$ & 229 & (52.0) & \\
\hline Viral pneumonia/influenza & 128 & $(20.3)$ & 33 & $(17.2)$ & 95 & (21.6) & \\
\hline Bronchiolitis & 84 & $(13.3)$ & 23 & $(12.0)$ & 61 & (13.9) & \\
\hline Other ${ }^{d}$ & 101 & $(16.0)$ & 46 & $(24.0)$ & 55 & $(12.5)$ & \\
\hline \multicolumn{8}{|l|}{ Acute Illness Severity } \\
\hline \multicolumn{7}{|l|}{ Increase in respiratory support by type ${ }^{e}$} & $<.001$ \\
\hline Initiate/increase oxygen flow or duration & 351 & $(55.5)$ & 53 & $(27.6)$ & 298 & $(67.7)$ & \\
\hline Initiate CPAP/BiPAP & 60 & (9.5) & 31 & $(16.1)$ & 29 & (6.6) & \\
\hline Increase pressure setting(s) or duration & 160 & (25.3) & 76 & (39.6) & 83 & (18.9) & \\
\hline Initiate full ventilation & 61 & $(9.7)$ & 32 & $(16.7)$ & 30 & $(6.8)$ & \\
\hline ICU admission & 255 & (40.4) & 100 & (52.1) & 155 & (35.2) & $<.001$ \\
\hline \multicolumn{8}{|c|}{${ }^{a} P$ value was determined using chi-square or Fisher's exact tests for categorical variables and Wilcoxon rank sum tests for continuous variables. } \\
\hline \multicolumn{8}{|c|}{ 'Dype of complex chronic conditions and technology dependence diagnostic coding categories are not mutually exclusive. } \\
\hline \multirow{2}{*}{\multicolumn{8}{|c|}{$\begin{array}{l}\text { 'Baseline respiratory support was defined as highest level of respiratory support required by a child when well prior to hospitalization as determined by electronic medical record review. } \\
\text { ¿Other ARI diagnosis type is any nonspecific diagnosis that might reflect an acute respiratory infection (see Appendix). }\end{array}$}} \\
\hline & & & & & & & \\
\hline \multirow{2}{*}{\multicolumn{8}{|c|}{$\begin{array}{l}\text { Increased respiratory support was defined as initiation of supplemental oxygen or increase in flow rate; initiation of CPAP, BiPAP, or full ventilation or increase in baseline settings; or increased } \\
\text { duration per day receiving these therapies that was required by the child at any time during hospitalization. } \\
\text { 'The majority (75.7\%) of hospitalizations with initiation/increase in oxygen were initiators with no baseline respiratory support. }\end{array}$}} \\
\hline & & & & & & & \\
\hline \multicolumn{8}{|c|}{ Abbreviations: ARI, acute respiratory infection; BiPAP, bilevel positive airway pressure; CPAP, continuous positive airway pressure; ICU, intensive care unit; IQR, interquartile } \\
\hline
\end{tabular}

Clinicians caring for children with $\mathrm{NI}$ hospitalized with ARI may find it difficult to determine a child's discharge readiness, in part because many children with $\mathrm{NI}$ have longer times to return to respiratory baseline and some never return to their 
TABLE 2. Unadjusted Analysis of Outcomes

\begin{tabular}{|c|c|c|c|c|c|c|c|}
\hline \multirow{2}{*}{$\begin{array}{l}\text { Unadjusted analysis } \\
\text { Primary outcome }(n, \%)\end{array}$} & \multicolumn{2}{|c|}{$\begin{array}{l}\text { Overall } \\
(\mathrm{N}=632)\end{array}$} & \multicolumn{2}{|c|}{$\begin{array}{l}\text { Discharged before return to } \\
\text { respiratory baseline } \\
(n=192)\end{array}$} & \multicolumn{2}{|c|}{$\begin{array}{l}\text { Discharged at respiratory baseline } \\
\qquad(\mathrm{n}=440)\end{array}$} & $\begin{array}{c}P \text { value }^{\mathrm{a}} \\
.81\end{array}$ \\
\hline & & & & & & & \\
\hline 30-day reutilization & 203 & $(32.1)$ & 63 & $(32.8)$ & 140 & $(31.8)$ & .71 \\
\hline 30-day hospital readmission & 165 & $(26.1)$ & 52 & $(27.1)$ & 113 & $(25.7)$ & .84 \\
\hline 30-day ED revisit & 38 & $(6.0)$ & 11 & $(5.7)$ & 27 & $(6.1)$ & \\
\hline 30-day hospital readmission & 120 & $(19.0)$ & 41 & $(21.4)$ & 79 & $(18.0)$ & .99 \\
\hline 30-day ED revisit & 16 & $(2.5)$ & 5 & $(2.6)$ & 11 & $(2.5)$ & \\
\hline LOS in days (median, [IQR]) & 4 & $(2,8)$ & 6 & $(3,11)$ & 4 & $(2,7)$ & $<.001$ \\
\hline
\end{tabular}

\section{TABLE 3. Adjusted Analysis of Outcomes}

\begin{tabular}{|c|c|c|}
\hline & Adjusted OR & $95 \% \mathrm{Cl}$ \\
\hline \multicolumn{3}{|l|}{ Primary outcome } \\
\hline 30-day reutilization & 0.80 & $0.51-1.26$ \\
\hline 30-day hospital readmission & 0.73 & $0.46-1.16$ \\
\hline 30-day ED revisit & 1.82 & $0.85-3.91$ \\
\hline \multicolumn{3}{|l|}{ Secondary outcome } \\
\hline 30-day reutilization for acute respiratory infection & 0.95 & $0.57-1.55$ \\
\hline 30-day hospital readmission & 0.89 & $0.54-1.46$ \\
\hline 30-day ED revisit & $-b$ & $-b$ \\
\hline
\end{tabular}

\section{TABLE 4. Subgroup Analysis Excluding Children Lost to Follow-up ${ }^{a}$}

\begin{tabular}{|c|c|c|c|c|}
\hline & Unadjusted OR & $95 \% \mathrm{Cl}$ & Adjusted $\mathbf{O R}^{\mathrm{b}}$ & $95 \% \mathrm{Cl}$ \\
\hline 30-day reutilization & 1.04 & $0.72-1.50$ & 0.82 & $0.52-1.28$ \\
\hline 30-day hospital readmission & 1.07 & $0.73-1.58$ & 0.70 & $0.43-1.13$ \\
\hline 30-day ED revisit & 0.91 & $0.44-1.88$ & 1.87 & $0.84-4.13$ \\
\hline
\end{tabular}

a Subgroup analysis excluded the 18 hospitalizations in which children were lost to follow-up ( $2.8 \%$ of the total cohort).

${ }^{b}$ Calculated using a generalized estimating equation to account for within-patient clustering and control for patient-level covariates (including age, race, ethnicity, insurance, number of complex chronic conditions, technology dependence [gastrointestinal, neurologic, respiratory], baseline respiratory support, primary respiratory illness diagnosis, increase in respiratory support, intensive care unit level care, and length of stay).

Abbreviations: ED, emergency department; $\mathrm{OR}$, odds ratio; $\mathrm{Cl}$, confidence interval.

pre-illness baseline. ${ }^{24}$ In otherwise healthy children hospitalized with respiratory infections such as pneumonia, discharge criteria typically include complete wean from respiratory support prior to discharge.,25 In our study's more complex children, whose parents already manage respiratory support at home, we hypothesize that discharging providers may be comfortable with discharge when the child has certain types of increased respiratory support compatible with home equipment, a par- ent skilled with monitoring the child's respiratory status, and the support of an experienced outpatient provider and home nursing providers. At our institution, outpatient respiratory support weans are primarily performed by pediatric pulmonologists and, for isolated weaning of supplemental oxygen or time using support, by parents and outpatient pediatricians.

Another important factor in determining a child's discharge readiness is the perspective of the child's parent. Berry et al 
found that children whose parents believe they are not healthy enough for discharge are more likely to experience unplanned hospital readmissions, ${ }^{24}$ signaling the role of child- and familyspecific factors in safe discharge decisions. Therefore, parents of children with $\mathrm{NI}$ should be proactively involved throughout the multidisciplinary discharge process, ${ }^{26,27}$ including the decision to discharge before return to respiratory baseline. Parents have identified ongoing provider support, opportunities to practice home care skills, and written instructions with contingency plans as important components of discharge readiness. ${ }^{28}$ Further work to create partnerships with these highly skilled caregivers in discharge decision making and transition planning are needed to promote safe discharge practices in this complex population.

In our study, children discharged before return to respiratory baseline were more likely to be older and privately insured compared with children discharged at respiratory baseline. Prior studies have found that social factors including low socioeconomic status influence ED provider admissions decisions for children with pneumonia. ${ }^{29,30}$ However, the role of socioeconomic factors in provider discharge decisions for children with $\mathrm{NI}$ has not been assessed. These traits may also be proxy markers of other sociodemographic factors, such as parent education level, financial hardship influencing ability to participate in a child's care at the bedside, access to comprehensive outpatient primary care, and availability of private home nursing. We hypothesize that these related characteristics directly and indirectly influence provider discharge decisions.

Discharging providers are likely more comfortable with discharge prior to return to respiratory baseline when the family has private duty nursing in the home. Home nurses can assist families in providing increased respiratory support and recognizing respiratory problems that may arise following discharge. However, home nursing shortages are common nationwide. ${ }^{31,32}$ Low-income children, children with respiratory technology use, and children without Medicaid have been found to have larger gaps in home nursing availability. ${ }^{31,32}$ Further studies are needed to understand the role of home nursing availability in provider discharge decisions in this population.

This study has several limitations. The retrospective design of this study creates the potential for residual confounding; there may be other clinical or demographic factors influencing hospital discharge decisions that we are unable to capture using EMR review, including parental knowledge and comfort managing illness, quality of discharge instructions, frequency of follow-up visits, and presence of skilled home nursing services. Categorization of children based on respiratory support status at discharge lends potential for misclassification of exposure; however, our substantial interrater reliability suggests that misclassification bias is small. This study's primary finding indicated no difference between exposure groups; although we may be unable to detect small differences, we had sufficient power with our sample size to detect meaningful differences in reutilization outcomes.

This study was not designed to capture outpatient time to return to respiratory baseline; prospective studies are needed to identify rates of return to respiratory baseline following ARI in children with NI. We did not measure the level of respiratory support used by children at the time of discharge and, therefore, are unable to estimate the amount of respiratory support weaning needed following discharge or the compatibility of support with home equipment using our data. In addition, this study focused on respiratory support modalities and, thus, did not measure inpatient utilization of mucociliary clearance technologies that might be hypothesized to decrease the time to return to baseline respiratory support. Next steps in evaluating treatment of ARI include investigating the effect of mucociliary clearance on both exposure and outcome in this population.

There may be other clinically meaningful outcomes for this population apart from reutilization that we have not assessed in this study, including increased respiratory support required following discharge, primary care reutilization, healthcare costs, or parent satisfaction with timing of and outcomes after discharge. Finally, although our hospital has reutilization rates for children with $\mathrm{NI}$ that are similar to other institutions in the United States, ${ }^{33}$ our results may not be generalizable to children with $\mathrm{NI}$ hospitalized at other institutions because local discharge processes and systems of care may be different. Prospective, multicenter investigation is needed to evaluate the clinical consequences of discharge before return to respiratory baseline more broadly.

\section{CONCLUSION}

At our institution, approximately one-quarter of children with $\mathrm{NI}$ hospitalized with ARI were discharged before return to respiratory baseline, but these children were not at increased risk of reutilization, compared with children discharged at respiratory baseline. Our findings suggest that return to baseline respiratory support might not be a necessary component of hospital discharge criteria. In otherwise clinically stable children with $\mathrm{NI}$, discharge before return to respiratory baseline may be reasonable if their parents are comfortable managing respiratory support at home.

\section{Acknowledgments}

The authors thank Jonathan Rodean of the Children's Hospital Association for his assistance with abstraction of PHIS data.

Disclosures: The authors have no conflicts of interest or financial relationships relevant to this article to disclose.

Funding: Dr Thomson was supported by the Agency for Healthcare Research and Quality under award number K08HS02513. Mses Chinchilla, Melink, and Tan were supported by the National Institutes of Health Medical Student Summer Research Fellowship in Pulmonary Diseases under award number 1T35HL113229-02. Dr Steuart received support for travel from Mead Johnson Nutrition. The Center for Clinical and Translational Science and Training at the University of Cincinnati in Ohio supported the use of the Research Electronic Data Capture (REDCap) online tools for data management.

\section{References}

1. Berry JG, Poduri A, Bonkowsky JL, et al. Trends in resource utilization by children with neurological impairment in the United States inpatient health care system: a repeat cross-sectional study. PLoS Med. 2012;9(1):e1001158. https://doi.org/10.1371/journal.pmed.1001158

2. Srivastava R, Jackson WD, Barnhart DC. Dysphagia and gastroesophageal reflux disease: dilemmas in diagnosis and management in children 
with neurological impairment. Pediatr Ann. 2010;39(4):225-231. https://doi org/10.3928/00904481-20100318-07

3. Seddon PC, Khan Y. Respiratory problems in children with neurological impairment. Arch Dis Child. 2003;88(1):75-78. https://doi.org/10.1136/adc.88.1.75

4. White $C M$, Statile $A M$, White $D L$, et al. Using quality improvement to optimise paediatric discharge efficiency. BMJ Qual Saf. 2014;23(5):428-436. https://doi.org/10.1136/bmjqs-2013-002556

5. Leyenaar JK, Lagu T, Shieh MS, Pekow PS, Lindenauer PK. Management and outcomes of pneumonia among children with complex chronic conditions. Pediatr Infect Dis J. 2014;33(9):907-911. https://doi.org/10.1097/ INF.0000000000000317

6. Stagliano DR, Nylund CM, Eide MB, Eberly MD. Children with Down syndrome are high-risk for severe respiratory syncytial virus disease. J Pediatr. 2015;166(3):703-709.e702. https://doi.org/10.1016/j.jpeds.2014.11.058

7. Kaiser SV, Bakel LA, Okumura MJ, Auerbach AD, Rosenthal J, Cabana MD. Risk factors for prolonged length of stay or complications during pediatric respiratory hospitalizations. Hosp Pediatr. 2015;5(9):461-473. https://doi. org/10.1542/hpeds.2014-0246

8. Landis JR, Koch GG. The measurement of observer agreement for categorical data. Biometrics. 1977;33(1):159-174.

9. Harris PA, Taylor R, Thielke R, Payne J, Gonzalez N, Conde JG. Research electronic data capture (REDCap)—a metadata-driven methodology and workflow process for providing translational research informatics support. J Biomed Inform. 2009;42(2):377-381. https://doi.org/10.1016/j. jbi.2008.08.010

10. Thomson JE, Feinstein JA, Hall M, Gay JC, Butts B, Berry JG. Identification of children with high-intensity neurological impairment. JAMA Pediatr. 2019. https://doi.org/10.1001/jamapediatrics.2019.2672

11. Feudtner C, Christakis DA, Connell FA. Pediatric deaths attributable to complex chronic conditions: a population-based study of Washington state, 1980-1997. Pediatrics. 2000;106(1 Pt 2):205-209.

12. Feudtner C, Hays RM, Haynes G, Geyer JR, Neff JM, Koepsell TD. Deaths attributed to pediatric complex chronic conditions: national trends and implications for supportive care services. Pediatrics. 2001;107(6):e99. https:// doi.org/10.1542/peds.107.6.e99

13. Feudtner C, Christakis DA, Zimmerman FJ, Muldoon JH, Neff JM, Koepsell TD. Characteristics of deaths occurring in children's hospitals: implications for supportive care services. Pediatrics. 2002;109(5):887-893. https://doi. org/10.1542/peds.109.5.887

14. Feudtner C, Feinstein JA, Zhong W, Hall M, Dai D. Pediatric complex chronic conditions classification system version 2: updated for ICD-10 and complex medical technology dependence and transplantation. BMC Pediatr. 2014;14:199. https://doi.org//10.1186/1471-2431-14-199

15. Berry JG HD, Kuo DZ, Cohen E, et al. Hospital utilization and characteristics of patients experiencing recurrent readmissions within children's hospitals. JAMA. 2011;305(7):682-690. https://doi.org/10.1001/jama.2011.122

16. Feudtner C, Villareale NL, Morray B, Sharp V, Hays RM, Neff JM. Technologydependency among patients discharged from a children's hospital: a retrospective cohort study. BMC Pediatr. 2005:5(1):8. https://doi.org/10.1186/1471-2431-5-8

17. Balamuth $F$, Weiss $S L$, Hall $M$, et al. Identifying pediatric severe sepsis and septic shock: accuracy of diagnosis codes. J Pediatr. 2015;167(6):1295-1300. e4. https://doi.org/10.1016/j.jpeds.2015.09.027

18. Kosecoff J, Kahn KL, Rogers WH, et al. Prospective payment system and impairment at discharge. The 'quicker-and-sicker' story revisited. JAMA. 1990:264(15):1980-1983.
19. Qian X, Russell LB, Valiyeva E, Miller JE. "Quicker and sicker" under Medicare's prospective payment system for hospitals: new evidence on an old issue from a national longitudinal survey. Bull Econ Res. 2011;63(1):1-27. https://doi.org/10.1111/j.1467-8586.2010.00369.x

20. Halm EA, Fine MJ, Marrie TJ, et al. Time to clinical stability in patients hospitalized with community-acquired pneumonia: implications for practice guidelines. JAMA. 1998;279(18):1452-1457. https://doi.org/10.1001/ jama.279.18.1452

21. Halm EA, Fine MJ, Kapoor WN, Singer DE, Marrie TJ, Siu AL. Instability on hospital discharge and the risk of adverse outcomes in patients with pneumonia. Arch Intern Med. 2002;162(11):1278-1284. https://doi.org/10.1001/ archinte.162.11.1278

22. Wolf RB, Edwards K, Grijalva CG, et al. Time to clinical stability among children hospitalized with pneumonia. J Hosp Med. 2015;10(6):380-383. https:// doi.org/10.1002/jhm.2370

23. Capelastegui A, España PP, Bilbao A, et al. Pneumonia: criteria for patient instability on hospital discharge. Chest. 2008;134(3):595-600. https://doi. org/10.1378/chest.07-3039

24. Berry JG, Ziniel SI, Freeman L, et al. Hospital readmission and parent perceptions of their child's hospital discharge. Int J Qual Health Care. 2013;25(5):573581. https://doi.org/10.1093/intahc/mzt051

25. Bradley JS, Byington CL, Shah SS, et al. The management of communityacquired pneumonia in infants and children older than 3 months of age: clinical practice guidelines by the Pediatric Infectious Diseases Society and the Infectious Diseases Society of America. Clin Infect Dis. 2011;53(7):e25-76. https://doi.org/10.1093/cid/cir531

26. Statile AM, Schondelmeyer AC, Thomson JE, et al. Improving discharge efficiency in medically complex pediatric patients. Pediatrics. 2016;138(2):e20153832. https://doi.org/10.1542/peds.2015-3832

27. Desai AD, Popalisky J, Simon TD, Mangione-Smith RM. The effectiveness of family-centered transition processes from hospital settings to home: a review of the literature. Hosp Pediatr. 2015;5(4):219-231. https://doi.org10.1542/ hpeds.2014-0097

28. Desai AD, Durkin LK, Jacob-Files EA, Mangione-Smith R. Caregiver perceptions of hospital to home transitions according to medical complexity: a qualitative study. Acad Pediatr. 2016;16(2):136-144. https://doi.org/10.1016/j. acap.2015.08.003

29. Agha MM, Glazier RH, Guttmann A. Relationship between social inequalities and ambulatory care-sensitive hospitalizations persists for up to 9 years among children born in a major Canadian urban center. Ambul Pediatr. 2007;7(3):258-262. https://doi.org/10.1016/j.ambp.2007.02.005

30. Flores G, Abreu M, Chaisson CE, Sun D. Keeping children out of hospitals: parents' and physicians' perspectives on how pediatric hospitalizations for ambulatory care-sensitive conditions can be avoided. Pediatrics. 2003;112(5):1021-1030. https://doi.org/10.1542/peds.112.5.1021

31. Weaver MS, Wichman B, Bace $S$, et al. Measuring the impact of the home health nursing shortage on family caregivers of children receiving palliative care. J Hosp Palliat Nurs. 2018;20(3):260-265. https://doi.org/10.1097/ NJH.0000000000000436

32. Leonard BJ, Brust JD, Sielaff BH. Determinants of home care nursing hours for technology-assisted children. Public Health Nurs. 1991;8(4):239-244. https://doi.org/10.1111/j.1525-1446.1991.tb00663.x

33. Cohen E, Berry JG, Camacho X, Anderson G, Wodchis W, Guttmann A. Patterns and costs of health care use of children with medical complexity. Pediatrics. 2012;130(6):e1463-1470. https://doi.org/10.1542/peds.2012-0175 